\title{
Key Concepts in the Salutogenic Model of Health
}

\author{
Monica Eriksson
}

Part II starts with a description of the theoretical framework of the salutogenic model of health, with special focus on the key concepts and reflection on the ontological and epistemological background of the health model, which so far is little explored and described (Chap. 9). Essential in the salutogenic model of health is the understanding of health as a process in a continuum, the health ease/dis-ease continuum. This is described and explained in the chapter. Some evidence of the relationship between sense of coherence (SOC) and health is presented.

In Chap. 10, the focus is on how the SOC influences stressor appraisal, positively as well as negatively. The processes of stimulus appraisal have a central place in salutogenic theory, even if they have received relatively little theoretical and empirical attention since Aaron Antonovsky's extensive treatment of stimulus appraisal in Unraveling the mystery of health: How people manage stress and stay well. The chapter aims to elevate researchers' appreciation of stimulus appraisal as Antonovsky's little-tested answer to three key questions: How does the SOC concept link to coping behavior, what is the mechanism that makes the connection, and what is the black box in between?

In Chap. 11, measurement issues are addressed concerning Antonovsky's original SOC questionnaires of 29 items and of 13 items, as well as several modified translations applicable at the individual, the family, the organization, and the community levels. Validity (face, construct, consensual, criterion, and predictive) and reliability issues (test-retest and internal consistency) of the scales are discussed. Criticism of the original scales is deliberated.

Chapter 12 presents and discusses theoretical considerations and empirical findings regarding the concepts: generalized resistance resources (GRRs) and generalized

\section{Eriksson $(\bowtie)$}

Department of Health Sciences, Section of Health Promotion and Care Sciences, Center on Salutogenesis, University West,

Trollhättan, Sweden

e-mail: monica.eriksson@hv.se resistance deficits (GRDs). Recent research findings are presented, showing how these resources or deficits impact the SOC. Suggestions for future research directions (e.g., individuals' differential susceptibility to environmental effects and eudaimonia/hedonia perspectives) and interventional implications are presented.

Chapter 13 discusses conceptual and concrete differences between generalized and specific resistance resources in the salutogenic model of health. It is important to distinguish between the two types of resistance resources to ensure that health promotion pays attention to both types. Specific resistance resources have as much or more relevance to health promotion practice as do generalized resistance resources. By drawing attention to the nature of specific resistance resources, one also draws attention to what should be a main aim of health promotion.

The part editor has devoted herself not only to producing overviews of salutogenic research but also to deepening knowledge of how the theory and the model of health developed subsequent to Antonovsky's seminal contributions. Salutogenesis has become the air I breathe! Certain trends in salutogenic research are evident:

- The translation and validation of the SOC questionnaires to languages other than English.

- The use of the SOC questionnaires in studies with an ever widening range of endpoints.

- An increasing appreciation that the salutogenic model has utility at the level of social theory (something more than a model).

- A growing interest in developing interventions aiming to strengthen the SOC among patients and professionals.

- The use of SOC questionnaires in evaluating the effectiveness of interventions.

Perhaps the most obvious trend is the burgeoning interest in salutogenic research as judged by the expanding literature. A search in PubMed (January 2020) using the terms salutogenesis and/or sense of coherence identified about 3000 pub- 
lished papers. Searching in the largest online library, WorldCat, more than 1200 doctoral dissertations on salutogenesis were identified. Most of the research effort has included a focus on the measurement of the SOC using the long and short versions of Antonovsky's Orientation to Life Questionnaire in a broad range of patient groups, community samples, and samples of age groups from young to old. As interest in using the SOC questionnaires has steadily increased, they have been translated into many different languages worldwide (see Chap. 11, Fig. 11.2), and this trend continues today.

The SOC questionnaires are used today in a much wider range of research areas compared to the early years. Examples of new areas are oral health, health behavior, and work and organizational life.

A particularly welcoming trend is the tendency to move from only measuring the SOC to applying salutogenic principles in health promotion practice, and in programs and interventions in various community settings.
Also notable is the trend in recent years to use SOC questionnaires to evaluate the effectiveness of interventions and health promotion programs. In many studies, an increase in SOC scores has been interpreted as due to successful intervention. Such conclusions are overly not really warranted as yet because we do not know the degree to which an increase in the magnitude of SOC scores may be due in part to normal fluctuations in the SOC, or other as-yet-unmeasured confounding factors.

There is much progress, but still, much work to do. There is an urgent need for new salutogenic instruments measuring human resources, which in turn could contribute to the development of the theory. Salutogenesis can be seen as an umbrella concept, and here a completely new world opens up to explore the relationship between related concepts and the SOC. Finally, educational sciences have the challenge to explore how an education approach can foster the strengthening of the SOC at the levels of the individual and in social groups.

Open Access This chapter is licensed under the terms of the Creative Commons Attribution 4.0 International License (http://creativecommons. org/licenses/by/4.0/), which permits use, sharing, adaptation, distribution and reproduction in any medium or format, as long as you give appropriate credit to the original author(s) and the source, provide a link to the Creative Commons license and indicate if changes were made.

The images or other third party material in this chapter are included in the chapter's Creative Commons license, unless indicated otherwise in a credit line to the material. If material is not included in the chapter's Creative Commons license and your intended use is not permitted by statutory regulation or exceeds the permitted use, you will need to obtain permission directly from the copyright holder. 